\title{
Cross-Link Formation between Mutant Galectins of Caenorhabditis elegans with a Substituted Cysteine Residue and Asialofetuin via a Photoactivatable Bifunctional Reagent
}

\author{
Mayumi TAmura, ${ }^{a}$ Tomoharu TAKeuchi,${ }^{a}$ Takamasa NonAKa, ${ }^{b}$ Ken-ichi Kasai,${ }^{c}$ and \\ Yoichiro ARATA*,a \\ ${ }^{a}$ Faculty of Pharmaceutical Sciences, Josai University; 1-1 Keyakidai, Sakado, Saitama 350-0295, Japan: ${ }^{b}$ School of \\ Pharmacy, Iwate Medical University; 2-1-1 Nishitokuta, Yahaba, Iwate 028-3694, Japan: and ${ }^{c}$ Department of Biological \\ Chemistry, Faculty of Pharmaceutical Sciences, Teikyo University; Sagamiko, Kanagawa 199-0195, Japan. \\ Received March 17, 2011; accepted March 22, 2011; published online April 6, 2011
}

LEC-1 is the first tandem repeat-type galectin isolated from an animal system; this galectin has two carbohydrate recognition domains in a single polypeptide chain. Because its two lectin domains have different sugarbinding profiles, these domains are thought to interact with different carbohydrate ligands. In our previous study, we showed that a mutant of LEC-1 in which a cysteine residue was introduced at a unique position in the $\mathrm{N}$-terminal lectin domain (Nh) can be cross-linked with a model glycoprotein ligand, bovine asialofetuin, by using a bifunctional photoactivatable cross-linking reagent, benzophenone-4-maleimide. In the present work, we applied the same procedure to the C-terminal lectin domain (Ch) of LEC-1. Cross-linked products were formed in the cases of two mutants in which a cysteine residue was introduced at $\mathrm{Lys}^{177}$ and $\mathrm{Ser}^{268}$, respectively. This method is very useful for capturing and assigning endogenous ligand glycoconjugates with relatively low affinities to each carbohydrate recognition domain of the whole tandem repeat-type galectin molecule.

Key words galectin; LEC-1; ligand; cross-link; maleimide; benzophenone

Galectins are a group of animal lectins characterized by their specificity for $\beta$-galactosides and an evolutionarily conserved sequence motif in the carbohydrate-binding site. Galectins are involved in a wide variety of biological phenomena, including development, cell differentiation, tumor metastasis, apoptosis, RNA splicing, and regulation of immune functioning. ${ }^{1-6)}$ Galectins can be classified into three types on the basis of their molecular architecture: proto, chimera, and tandem-repeat types. LEC-1, the $32-\mathrm{kDa}$ galectin of the nematode, Caenorhabditis elegans ( $C$. elegans), was the first example of a tandem repeat-type galectin composed of two homologous regions. ${ }^{7,8)}$

A variety of pyridylaminated oligosaccharides were used as analytes in frontal affinity chromatography, ${ }^{9)}$ and the result showed that the two lectin domains of LEC-1 have different sugar-binding profiles. ${ }^{10)}$ This finding suggests that the endogenous ligand glycoconjugates for the two domains of LEC-1 differ. However, it is difficult to confirm this finding, especially when the binding ability of each domain is not adequately strong. We developed a new approach to overcome this problem. We prepared LEC-1 mutants in which a cysteine residue was introduced near the binding site of the $\mathrm{N}$ terminal half domain; after incubation with a model glycoprotein ligand, asialofetuin, cross-linked products were formed using a photoactivatable bifunctional reagent, benzophenone-4-maleimide (BPM). Among the five LEC-1 mutants produced, one of them (LEC-1 Q38C) produced the cross-linked product. ${ }^{11)}$ This method was also applied to human galectin-1 (Gal-1). At first, all cysteine residues were substituted with Ser residues, and Lys ${ }^{28}$ of the resultant cysteine-less Gal-1 was substituted with a cysteine residue. The position of $\mathrm{Lys}^{28}$ corresponds to Gln ${ }^{38}$ of LEC-1. Crosslinked products between hGal-1 K28C and asialofetuin via BPM were observed. ${ }^{12)}$ In the present study, we produced several LEC-1 mutants, each with a cysteine substitution in the C-terminal lectin domain (Ch) to detect interactions with asialofetuin. Two mutants, K177C and S268C, produced cross-linked products. This procedure is very promising for capturing and identifying endogenous ligands for each lectin domain of the tandem repeat-type galectin, LEC-1.

\section{MATERIALS AND METHODS}

Chemicals Rabbit anti-bovine fetuin polyclonal antibody was purchased from Chemicon International (Temecula, CA, U.S.A.). BPM and bovine fetuin were purchased from Sigma (St. Louis, MO, U.S.A.). Sialidase and $\beta$-galactosidase were purchased from Seikagaku Co. (Tokyo, Japan).

Construction of Mutant Lectin Genes A cysteine residue was introduced using site-directed mutagenesis of LEC-1 cDNA (LEC-1 contains no cysteine residues). ${ }^{8}$ Residues subjected to substitution were selected on the basis of the X-ray crystal structure of LEC-1 in complex with galactose. $^{13)}$ To facilitate cross-link formation, hydrophilic amino acid residues with side chains extending from the surface of the protein toward the bound galactose but not contacting it were selected. Site-directed mutagenesis was performed as described previously. ${ }^{11,12}$ ) The following oligonucleotide primers were used for mutagenesis (substitution sites are underlined): E153C, 5'-ACCCGGTACCATACTGCAGCGGACTCGCCAACGGAC-3'; K177C, 5'-GTTGAGAAGAAGGCATGCCGCTTCCACGTCAACCTTCTCCGC-3'; D199C, 5'-TCCACTTCAACCCGCGGTTCTGTGAGAAGCACGTCATC-3'; N211C, 5' -AACTCTCTTGCCGC $\underline{\text { ATGC- }}$ GAATGGGGAAATGAGGAGCGCGAGGGA-3'; and

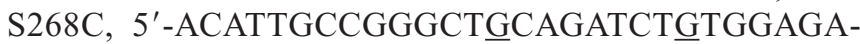
CATTGAGCTGTCTGGAA-3'.

Purification of Recombinant Galectins Recombinant proteins were expressed and purified as described previously. ${ }^{11,12)}$ 
Preparation of Asialofetuin for Cross-Linking Bovine fetuin was treated with sialidase to remove the sialic acid attached to the non-reducing galactose end residues of the sugar chain. The resultant asialofetuin was purified as described previously ${ }^{14)}$ and used as a model glycoprotein ligand for LEC-1.

Covalent Cross-Linking of Mutated LEC-1 Proteins to Asialofetuin Cysteine-introduced LEC-1 was cross-linked to the model glycoprotein ligand, asialofetuin, as described previously. ${ }^{11,12,14)}$ In brief, purified recombinant LEC-1 or the single cysteine-containing mutant of LEC-1 was dialyzed against phosphate-buffered saline (PBS) containing $1 \mathrm{~mm}$ ethylenediaminetetraacetic acid (EDTA) (pH 7.2) (EDTAPBS). BPM was dissolved in dimethylformamide, and the solution was added to the lectin solution (final concentration, $1 \mu \mathrm{M}$ BPM). For negative controls, dimethylformamide was added instead of the BPM solution. After incubation in the dark for $30 \mathrm{~min}$ at $23^{\circ} \mathrm{C}$, unreacted BPM was quenched by the addition of dithiothreitol (final concentration, $20 \mathrm{~mm}$ ). Asialofetuin in EDTA-PBS was added, and the reaction mixture was irradiated with a long-wavelength ultraviolet light for $3 \mathrm{~min}$ at $4{ }^{\circ} \mathrm{C}$. The cross-linked products were analyzed by western blotting using a rabbit polyclonal antibody against $\mathrm{LEC}-1^{8)}$ and a rabbit polyclonal antibody against fetuin.

\section{RESULTS}

Construction of LEC-1 Mutants Containing Substituted Cysteine Residues In the current study, we investigated whether the model glycoprotein ligand asialofetuin is actually recognized by the C-terminal lectin domains of LEC-1 mutants that have a substituted cysteine residue near the binding site of the C-terminal lectin domain and whether they can be cross-linked to asialofetuin via BPM.

Each of the cysteine-substituted mutants was adsorbed to the asialofetuin-Sepharose and could be eluted with $0.1 \mathrm{M}$ lactose. This confirms that the mutants retained their binding ability (data not shown). The purity of each recombinant protein was checked by sodium dodecyl sulfate-polyacrylamide gel electrophoresis (SDS-PAGE). In the absence of other bands with higher molecular weights, if any band appeared in the higher-molecular-weight region after the cross-linking reaction, it was expected that the formation of cross-linked complexes would be easily detected.

Cross-Linking of Mutant LEC-1s to Asialofetuin BPM primarily reacts with the introduced sulfhydryl group in the binding site region of the mutant LEC-1 via its maleimide moiety. ${ }^{15)}$ Ultraviolet irradiation activates the benzophenone group at the other terminal, which can form covalent bonds with nearby groups. Therefore, if lectin binds a glycoprotein molecule, a covalently linked complex that can be easily detected on SDS-PAGE can be formed. The fact that no species with higher molecular mass were observed in a control experiment wherein wild-type LEC-1 was used suggests that although asialofetuin contains several cysteine residues that can react with the maleimide group of BPM, no cross-link formation occurred with the wild-type LEC-1. Therefore, any cross-linked products observed in the experiments using mutant LEC-1 should be attributable to BPM reacting with the introduced cysteine residues.
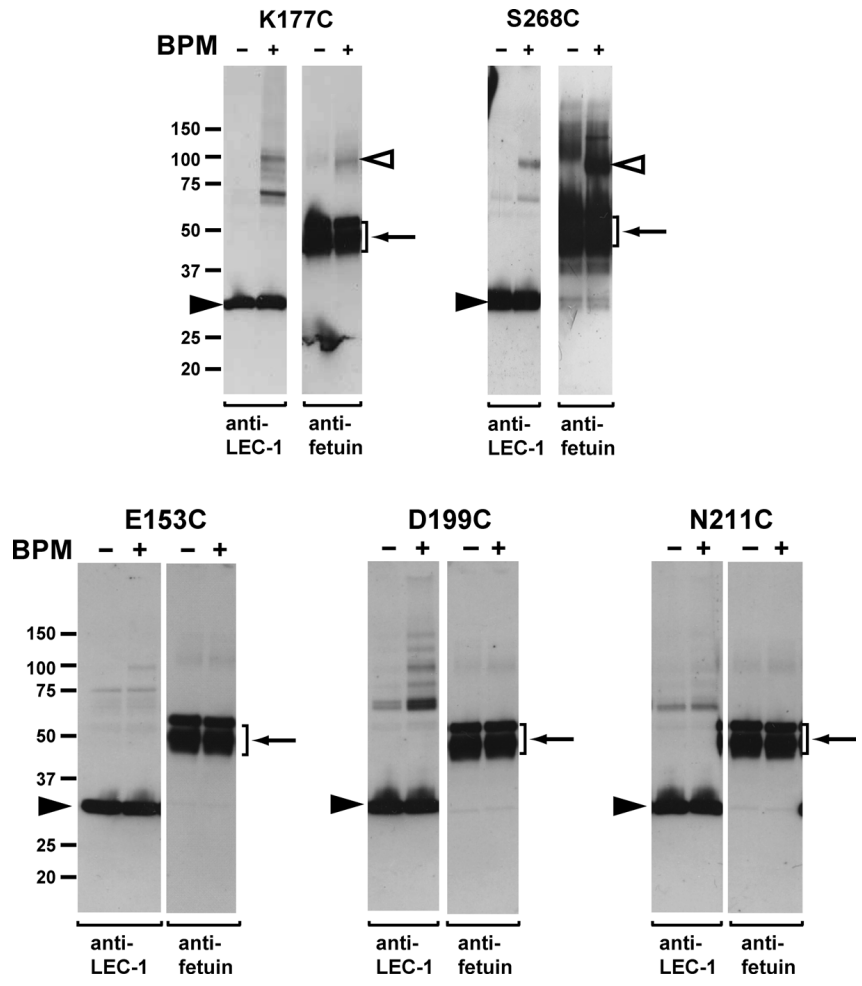

Fig. 1. Cross-Linking of LEC-1 Mutants and Asialofetuin

LEC-1 mutants with a substituted cysteine residue were incubated in the presence $(+)$ or absence $(-)$ of BPM in the dark and then irradiated with UV light in the presence of asialofetuin. The products were then analyzed by western blotting. A new band with a molecular mass of $90 \mathrm{kDa}$ was detected with antiserum against LEC-1 in mutants K177C and S268C, but not in the remaining mutants E153C, D199C, and N211C. The $90-\mathrm{kDa}$ band was also detected with anti-fetuin antiserum. The molecular masses of marker proteins are indicated on the left. The black arrowhead shows the position of the LEC-1 mutants; the positions of asialofetuin (arrow) and the expected positions of $\mathrm{K} 177 \mathrm{C} / \mathrm{S} 268 \mathrm{C}$-asialofetuin heterodimers (white arrowheads) are shown on the right.

Five LEC-1 mutants containing one cysteine residue each at $\mathrm{Glu}^{153}$ (E153C), Lys ${ }^{177}$ (K177C), Asp ${ }^{199}$ (D199C), Asn ${ }^{211}$ (N211C), or $\operatorname{Ser}^{268}$ (S268C) were reacted with BPM in the dark, mixed with asialofetuin, irradiated with ultraviolet light, and analyzed by western blotting. As shown in Fig. 1, the antibody against LEC-1 recognized an additional major band with a molecular mass of approximately $90 \mathrm{kDa}$ (white arrowhead in Fig. 1) in the cases of mutants K177C and $\mathrm{S} 268 \mathrm{C}$ in the presence of BPM. The observed molecular mass of approximately $90 \mathrm{kDa}$ is consistent with that of the cross-linked complex. In contrast, no corresponding highermolecular-mass bands were detected in the cases of mutants E153C, D199C, and N211C, also shown in Fig. 1.

Effect of Lactose on Cross-Linking As shown in Fig. 2 , in the presence of $0.1 \mathrm{M}$ lactose, cross-linking between asialofetuin and mutants K177C and S268C was remarkably reduced. These results suggest that cross-linking occurs only when a specific lectin-sugar interaction exists. Furthermore, no cross-linked products were observed when asialofetuin treated with Streptococcus $6646 \mathrm{~K} \beta$-galactosidase was used instead of asialofetuin (data not shown). This indicates that the removal of terminal galactose moieties from glycoproteins results in the loss of specific interaction. 


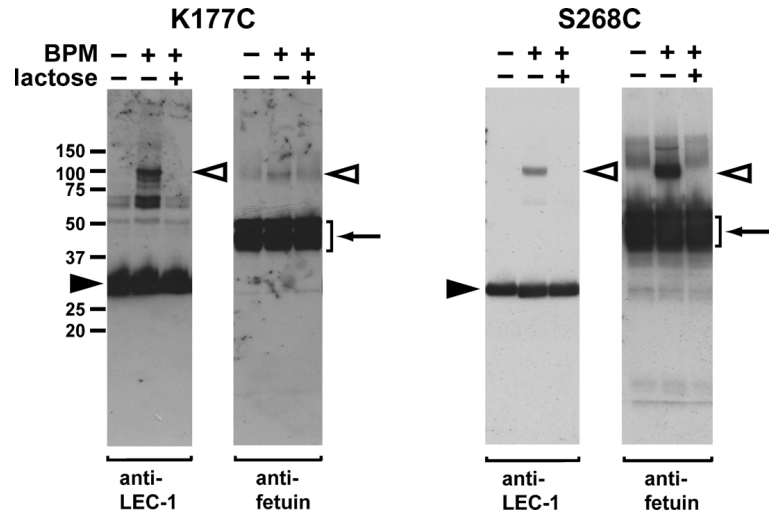

Fig. 2. Inhibition of Cross-Linking between the Mutants K177C and S268C with Asialofetuin by Lactose

The cross-linking of $\mathrm{K} 177 \mathrm{C}$ and $\mathrm{S} 268 \mathrm{C}$ with asialofetuin was significantly reduced in the presence of $0.1 \mathrm{~m}$ lactose in the reaction mixture. The black arrowhead shows the position of the LEC-1 mutants; the positions of asialofetuin (arrow) and the expected positions of $\mathrm{K} 177 \mathrm{C} / \mathrm{S} 268 \mathrm{C}$-asialofetuin heterodimers (white arrowheads) are shown on the right.

\section{DISCUSSION}

In the present study, two LEC-1 mutants with a cysteine residue introduced into the C-terminal lectin domain (at positions Lys ${ }^{177}$ or $\mathrm{Ser}^{268}$ ) gave rise to cross-linked products with the model glycoprotein ligand, asialofetuin, via BPM.

According to the X-ray crystallographic analysis of LEC-1 in a complex with galactose, ${ }^{13)}$ Lys $^{177}$ in the C-terminal lectin domain $(\mathrm{Ch})$, one of the positions where the introduction of a cysteine residue resulted in cross-linking with asialofetuin via BPM, corresponds to $\mathrm{Gln}^{38}$ in the N-terminal lectin domain (Nh), which was previously shown to yield a similar result. ${ }^{11)}$ Furthermore, a similar result was obtained when $\mathrm{Lys}^{28}$ of human galectin-1 (Gal-1), which corresponds to $\mathrm{Gln}^{38}$ of $\mathrm{Nh}$ of LEC-1 and Lys ${ }^{177}$ of Ch of LEC-1, which was substituted with a cysteine residue. ${ }^{12)}$ These positions have proved to be suitable for substitution by a cysteine residue to form crosslinks and for identifying endogenous glycoprotein ligands in future experiments. On the other hand, Ser $^{268}$ of LEC-1 was shown to be a new position for cysteine substitution.

Although the $\mathrm{Gal} \beta 1 \rightarrow 4 \mathrm{GlcNAc}$ ( $N$-acetyllactosamine) disaccharide unit is thought to be the endogenous glyco-epitope recognized by vertebrate galectins and the galectins of protostomia species such as $C$. elegans also bind to the same disaccharide structure, the existence of glycan-containing $\mathrm{N}$ acetyllactosamine units has not been confirmed in protostomia. ${ }^{16-21)}$ Recently, we found that another $C$. elegans galectin LEC-6 strongly interacts with $N$-glycans containing a Gal-Fuc disaccharide unit attached at position 6 of the innermost GlcNAc residue. ${ }^{22)}$ We also found that LEC-6 interacts with $\mathrm{Gal} \beta 1 \rightarrow 4$ Fuc more strongly than does $\mathrm{Gal} \beta 1 \rightarrow$ $3 \mathrm{Fuc}^{23)}$ by using chemically synthesized Gal $\beta 1 \rightarrow 4 \mathrm{Fuc}$ and Gal $\beta 1 \rightarrow 3$ Fuc. $^{24)}$ Furthermore, the tandem repeat-type nematode galectin LEC-1 also shows preferential binding to Gal $\beta 1 \rightarrow 4$ Fuc. $^{23)}$ However, the two domains of LEC- 1 exhibited different binding profiles when examined with $\mathrm{Gal} \beta 1 \rightarrow$ 4Fuc, Gal $\beta 1 \rightarrow 3$ Fuc, and $\mathrm{Gal} \beta 1 \rightarrow 4 \mathrm{GlcNAc}$. Furthermore, both domains have significantly low affinity for LEC-6-binding endogenous glycans. This suggests that endogenous ligands for LEC-1 differ from those for LEC- $6 .{ }^{25)}$
We previously performed a detailed analysis with a variety of fluorescence-labeled oligosaccharides in frontal affinity chromatography, and reported that the two lectin domains of LEC-1 have different sugar-binding profiles. ${ }^{10)}$ The method reported in this paper should enable us to capture and characterize independent endogenous ligand glycoconjugates for each binding site of a lectin with multiple binding sites, even if the interactions between the lectin and its ligand glycoconjugates are relatively low and no other procedure is applicable. LEC- 1 is considered to be bound to the $\beta$-galactoside structure of the endogenous ligand existing in the insoluble fraction because LEC-1 was extracted from C. elegans with lactose-containing buffer. ${ }^{7,8)}$ We are currently working toward isolating the endogenous ligand glycoproteins by adding the Cys-introduced LEC-1 mutant recombinant proteins preincubated with BPM to the insoluble fraction of $C$. elegans after lactose extraction to remove the bound endogenous galectins. Since cross-linking of LEC-1 mutants with their ligands via BPM and isolation from other proteins is applicable even when the isolation process requires severe conditions such as detergent treatment or use of denaturing agent while the interaction may be relatively weak, this procedure should enable us to isolate and assign the captured ligand glycoproteins by using mass spectrometry. The development of such methodology would certainly contribute greatly to understanding of the significance of carbohydrate recognition in biological systems.

Acknowledgements We thank Dr. Yoko Nemoto-Sasaki for helpful discussions.

\section{REFERENCES}

1) Kasai K., Hirabayashi J., J. Biochem., 119, 1-8 (1996)

2) Leffler H., Carlsson S., Hedlund M., Qian Y., Poirier F., Glycoconj. J., 19, 433-440 (2004).

3) Cooper D. N., Barondes S. H., Glycobiology, 9, 979—984 (1999).

4) Barondes S. H., Castronovo V., Cooper D. N. W., Cummings R. D., Drickamer K., Feizi T., Gitt M. A., Hirabayashi J., Hughes C., Kasai K., Leffler H., Liu F.-T., Lotan R., Mercurio A. M., Monsigny M., Pillai S., Poirer F., Raz A., Rigby P. W. J., Rini J. M., Wang J. L., Cell, 76, 597-598 (1994).

5) Liu F. T., Rabinovich G. A., Nat. Rev. Cancer, 5, 29-41 (2005).

6) Hernandez J. D., Baum L. G., Glycobiology, 12, 127R_-136R (2002).

7) Hirabayashi J., Satoh M., Ohyama Y., Kasai K., J. Biochem., 111, 553 -555 (1992).

8) Hirabayashi J., Satoh M., Kasai K., J. Biol. Chem., 267, 15485-15490 (1992).

9) Hirabayashi J., Arata Y., Kasai K., Methods Enzymol., 362, 353-368 (2003).

10) Arata Y., Hirabayashi J., Kasai K., J. Biol. Chem., 276, 3068-3077 (2001).

11) Arata Y., Tamura M., Nonaka T., Kasai K., Biochem. Biophys. Res. Commun., 350, 185-190 (2006).

12) Tamura M., Igarashi T., Kasai K., Arata Y., Biochem. Biophys. Res. Commun., 390, 581-584 (2009).

13) Nonaka T., Sekihashi K., Nishizaki S., Arata Y., Hirabayashi J., Kasai K., Mitsui Y., Glycoconj. J., 16, S119 (1999).

14) Tamura M., Igarashi T., Kasai K., Arata Y., Yakugaku Zasshi, 130, 1375-1379 (2010)

15) Dormán G., Prestwich G. D., Biochemistry, 33, 5661—5673 (1994).

16) Guerardel Y., Balanzino L., Maes E., Leroy Y., Coddeville B., Oriol R., Strecker G., Biochem. J., 357, 167-182 (2001).

17) Schachter H., Curr. Opin. Struct. Biol., 14, 607-616 (2004).

18) Cipollo J. F., Awad A. M., Costello C. E., Hirschberg C. B., J. Biol. Chem., 280, 26063-26072 (2005).

19) Griffitts J. S., Haslam S. M., Yang T., Garczynski S. F., Mulloy B., 
Morris H., Cremer P. S., Dell A., Adang M. J., Aroian R. V., Science, 307, 922-925 (2005).

20) Hanneman A. J., Rosa J. C., Ashline D., Reinhold V. N., Glycobiology, 16, 874-890 (2006).

21) Paschinger K., Gutternigg M., Rendic D., Wilson I. B., Carbohydr Res., 343, 2041-2049 (2008).

22) Takeuchi T., Hayama K., Hirabayashi J., Kasai K., Glycobiology, 18 $882-890$ (2008).
23) Takeuchi T., Nishiyama K., Sugiura K., Takahashi M., Yamada A., Kobayashi S., Takahashi H., Natsugari H., Kasai K., Glycobiology, 19 1503 - 1510 (2009)

24) Nishiyama K., Yamada A., Takahashi M., Takeuchi T., Kasai K., Kobayashi S., Natsugari H., Takahashi H., Chem. Pharm. Bull., 58, 495-500 (2010).

25) Takeuchi T., Sugiura K., Nishiyama K., Takahashi H., Natsugari H., Arata Y., Kasai K., Biol. Pharm. Bull., in press (2011). 